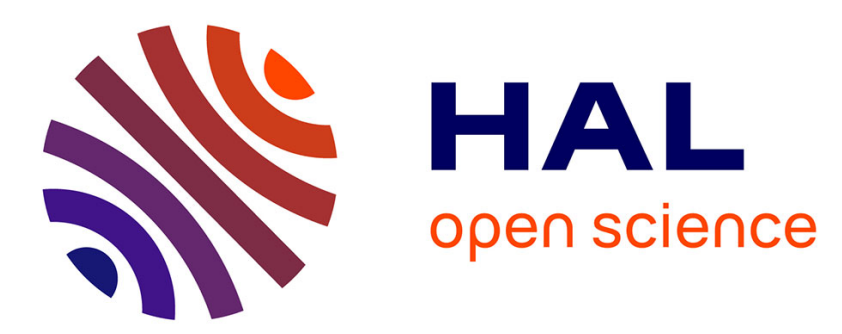

\title{
Néel temperature of a low-dimensional antiferromagnet in a magnetic field
}

\author{
J. Villain, J.M. Loveluck
}

\section{To cite this version:}

J. Villain, J.M. Loveluck. Néel temperature of a low-dimensional antiferromagnet in a magnetic field. Journal de Physique Lettres, 1977, 38 (2), pp.77-80. 10.1051/jphyslet:0197700380207700 . jpa-00231330

\section{HAL Id: jpa-00231330 https://hal.science/jpa-00231330}

Submitted on 1 Jan 1977

HAL is a multi-disciplinary open access archive for the deposit and dissemination of scientific research documents, whether they are published or not. The documents may come from teaching and research institutions in France or abroad, or from public or private research centers.
L'archive ouverte pluridisciplinaire HAL, est destinée au dépôt et à la diffusion de documents scientifiques de niveau recherche, publiés ou non, émanant des établissements d'enseignement et de recherche français ou étrangers, des laboratoires publics ou privés. 


\title{
NÉEL TEMPERATURE OF A LOW-DIMENSIONAL ANTIFERROMAGNET IN A MAGNETIC FIELD
}

\author{
J. VILLAIN \\ Laboratoire de Diffraction Neutronique, Département de Recherche Fondamentale, \\ Centre d'Etudes Nucléaires de Grenoble, 85 X, 38041 Grenoble Cedex, France

\section{J. M. LOVELUCK} \\ Institut Laue-Langevin, 156 X Centre de Tri, 38042 Grenoble Cedex, France \\ (Reçu le 25 octobre 1976, révisé le 16 décembre 1976, accepté le 21 décembre 1976)

\begin{abstract}
Résumé. - Un champ magnétique approprié peut augmenter la longueur de corrélation des chaînes antiferromagnétiques classiques de type Heisenberg ou XY. Il en résulte que la température de Néel des antiferromagnétiques presque unidimensionnels peut être augmentée par un champ magnétique, en accord avec des expériences récentes sur TMMC.
\end{abstract}

\begin{abstract}
A magnetic field of appropriate magnitude can increase the correlation length of onedimensional, classical antiferromagnets of the Heisenberg or XY type. It follows that the Néel temperature of quasi-one-dimensional antiferromagnets can be increased by a magnetic field, in agreement with recent experiments on TMMC.
\end{abstract}

1. Introduction. - Recent experiments [1] show that the Néel temperature $T_{\mathrm{N}}$ of the quasi-one-dimensional antiferromagnet $\left(\mathrm{CH}_{3}\right)_{4} \mathrm{NMnCl}_{3}$, also known as TMMC, is increased by $18 \%$ under a magnetic field of $10000 \varnothing$. The reduction of quantum fluctuations [2] fails [1] to explain the effect.

It will be shown in the present letter that the application of a magnetic field $H$ to a classical antiferromagnet can increase the Néel température whether the system has a sufficiently marked one-dimensional or two-dimensional character. The system will be assumed to be isotropic in the spin space for $H=0$. The present work applies to spins of dimensionality $n=2$ or 3 , and might be extended to higher values of $n$. The Ising case $n=1$ is excluded.

It is of interest to recall that in usual, three-dimensional antiferromagnets the Néel temperature decreases under a magnetic field. For instance, in the molecular field approximation :

$$
K_{\mathrm{B}} T_{\mathrm{N}}=2|J| z s^{2} \mathfrak{L}(h) / h
$$

where $2|J| z$ is the molecular field at $T=0, \mathcal{L}$ is the Langevin function and $h=s H / 2 K_{\mathrm{B}} T$.

For the sake of simplicity. the present work will be restricted to quasi-one-dimensional antiferromagnets ; the results for quasi-two-dimensional antiferromagnets can be obtained in a similar way and will be given without proof in section 5 .
2. The model. - Consider a 3-dimensional, periodic array of $N^{\prime}$ parallel chains. labelled by $i$. with $N$ classical, $n$-dimensional spins $\mathbf{S}_{i m}$ located on each chain with a uniform intersite distance $l$. The Hamiltonian is assumed to be :

$$
\begin{array}{r}
\mathscr{H}=-2 J \sum_{i, m} \mathbf{S}_{i m} \cdot \mathbf{S}_{i, m+l}-\sum_{i, j, m} J_{i j}^{\prime} \mathbf{S}_{i m} \cdot \mathbf{S}_{j m}- \\
-H \sum_{i, m} S_{i m}^{z}
\end{array}
$$

with $J<0$. $\left|\mathbf{S}_{i m}\right|=s, J_{i j}^{\prime}=J^{\prime}$ if chains $i$ and $j$ are nearest neighbours, otherwise $J_{i j}^{\prime}=0$.

If $J^{\prime}=0$, there is no long range ordering, and the correlations between the spin components $S_{i m}^{x}$ orthogonal to the magnetic field direction $z$ are given at long distances $m[3,4.5]$ by :

$$
\left\langle S_{i m^{\prime}}^{x} S_{j, m^{\prime}+m}^{x}\right\rangle=\delta_{i j}(-1)^{m} \exp -|m| / \xi .
$$

For $H=0$. this relation is exact for all values of $m$; at low temperature $T$. $\xi$ is given [3] by

$$
\begin{array}{ll}
\xi(T)=2|J| s^{2} / K_{\mathrm{B}} T & \text { for } n=3 \\
\xi(T)=4|J| s^{2} / K_{\mathrm{B}} T & \text { for } n=2 \\
\xi(T)=\frac{1}{2} \exp \left(4|J| s^{2} / K_{\mathrm{B}} T\right) & \text { for } n=1 .
\end{array}
$$

The calculation of $\xi$ in a field involves the calculation of two eigenvalues of the transfer operator; only 
the lowest eigenvalue has been calculated analytically for $n=3$ [4]. Numerical calculations have been performed [5], but it is of interest to give the simple, qualitative discussion which follows. In section 3, the effect of a magnetic field on an isolated chain $\left(J^{\prime}=0\right)$ will be considered, and the consequences for $J^{\prime} \neq 0$ will be studied in section 4 .

3. Isolated chain in a magnetic field at low temperature. - In this section, we argue that a sufficiently high magnetic field $H$ applied along $z$ to an isolated chain at low temperature $K_{\mathrm{B}} T \ll|J| s^{2}$. suppresses spin fluctuations along $z$; on the other hand. a very high field also suppresses fluctuations along the other directions. Therefore we shall assume :

$$
H \ll 4|J| s .
$$

It is easy to eliminate the odd sites in (1) and to obtain the free energy as a function of the even spins $\mathbf{S}_{i, 2 p}$ : this is the so-called decimation procedure; the essential result at low temperature is that the effective bilinear interaction between even spins is $|J| / 2$ instead of $-|J|$. and the magnetic field gives rise to an effective anisotropy :

$$
\tilde{H}_{\text {an }}=\sum_{i, p}\left(\mathbf{H} . \mathbf{S}_{i, 2 p}\right)^{2} / 8|J| s^{2} .
$$

There is also a negligible effective magnetic field and negligible effective biquadratic interactions.

Fluctuations along $z$ are suppressed when the order of magnitude of the anisotropy energy for a length of order $\xi$ is larger than $K_{\mathrm{B}} T$. i.e. :

$$
H^{2} \xi / 8|J| \gtrsim K_{\mathrm{B}} T
$$

or. according to $(2 a)$ and $(2 b)$ :

$$
K_{\mathrm{B}} T \lesssim H s .
$$

Comparing with (3). it is seen that a magnetic field which satisfies :

$$
K_{\mathrm{B}} T \ll H s \ll 4|J| s^{2}
$$

transforms an $\mathrm{XY}$ chain into an Ising chain, and a Heisenberg chain into an XY chain. In fact. this result is independent of the space dimensionality $d$.

3.1 Case of the Heisenberg chain $(n=3)$. The correlation length is given by $(2 a)$ in zero field; if (5) is satisfied. the system should behave as an XY chain since the spin component along $\mathbf{H}$ is always very small : therefore the correlation length is given by $(2 b)$ and is twice as large as in zero field. This value $2 \xi(0)$ is obviously a maximum : if the field is too small the spins recover their degree of freedom along $z$. and if the field is too large the spins progressively lose their degree of freedom along the other directions. The maximum is reached when condition (5) is satisfied; in addition, $\xi(H)$ is expected to be symmetric in $H$ and analytic at $H=0$; this gives the general shape of the curve $\xi(H)$.
More complete information can be obtained by the transfer matrix method [5], and we show in figure 1 typical results obtained for different temperatures. For these calculations we have adopted the definition of $\xi$ given in reference [5]. in terms of an expansion of the wave-vector dependent susceptibility about the zone-boundary. since this allows the extension to higher temperatures when $\xi$ is small. The calculated results, for low temperatures, corroborate the qualitative argument outlined above. For higher temperatures there is no increase of $\xi$ with field because condition (5) cannot be satisfied.

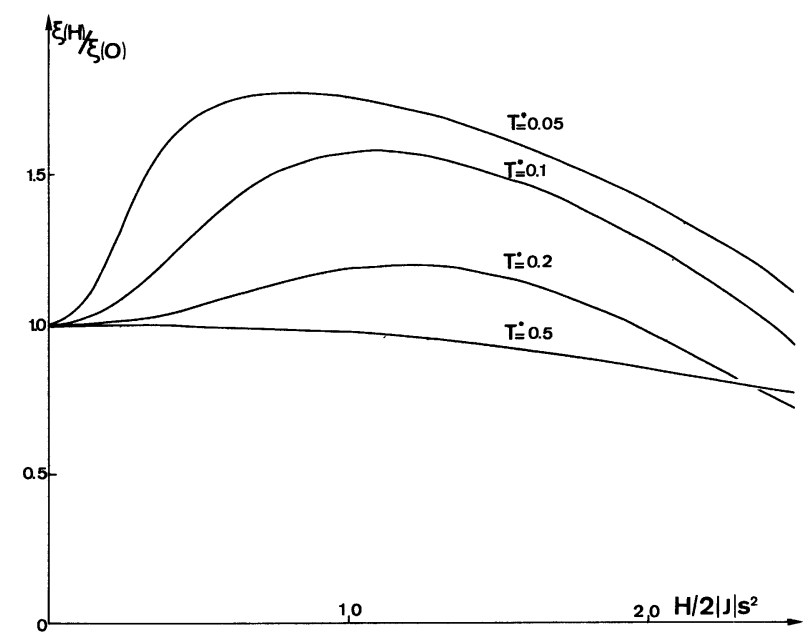

FIG. 1. - Calculated values of the ratio $\xi(H) / \xi(0)$ for a Heisenberg chain are shown as a function of $H / 2|J| s$ for a number of values of reduced temperature $\left.T^{*}=K_{\mathrm{B}} T / 2|J| s^{2}\right)$.

3.2 CASE OF THE XY ChaIN $(n=2)$. - A similar discussion shows that the correlation length. given by $(2 b)$ in zero field. is given by $(2 c)$ when condition (5) is fulfilled. In principle, the correlation length can be multiplied by an arbitrarily large factor when a magnetic field is applied at very low temperature.

4. Néel temperature of a quasi-one-dimensional antiferromagnet in a magnetic field. - For $H=0$, the Néel temperature $T_{\mathrm{N}}$ is given by the following equation. which is derived in the appendix :

$$
\left|J^{\prime}\right| s^{2} \xi\left(T_{\mathrm{N}}\right)=C_{n} K_{\mathrm{B}} T_{\mathrm{N}}
$$

where $\xi(T)$ is defined by (2) and $C_{n}$ is a coefficient which. for a given lattice, depends on the spin dimensionality $n$. In the mean field approximation [2], $C_{n}$ is independent of $n$.

Insertion of (2) into (6) yields :

$\left(\mid J^{\prime} s^{2} / 2 K_{\mathrm{B}} T_{\mathrm{N}}\right) \exp \left(4|J| s^{2} / K_{\mathrm{B}} T_{\mathrm{N}}\right)=C_{1}$

for $n=1$

$$
K_{\mathrm{B}} T_{\mathrm{N}}=2 s^{2} \sqrt{2\left|J J^{\prime}\right| / C_{2}}
$$

for $n=2$

$$
K_{\mathrm{B}} T_{\mathrm{N}}=2 s^{2} \sqrt{\left|J J^{\prime}\right| / C_{3}}
$$

for $n=3$ 


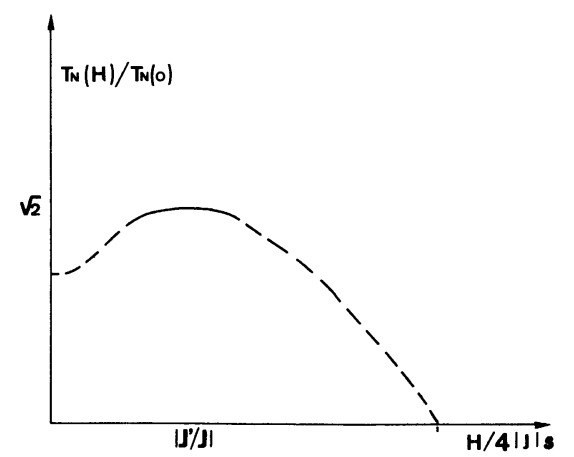

Fig. 2. - Typical behaviour of $T_{\mathrm{N}}$ as a function of $H$ for a quasione-dimensional, Heisenberg antiferromagnet. Dashed parts are interpolated.

We shall now consider the effect of a field satisfying (5) : for an XY chain $(n=2)$. $T_{\mathrm{N}}$ is given by $(7 a)$ in the presence of this field. and. if $\left|J^{\prime}\right|$ is sufficiently small, this value is always higher than the zero-field Néel temperature. given by $(7 b)$.

The case of the Heisenberg chain $(n=3)$ requires more discussion : the maximum value of $T_{\mathrm{N}}$. in a field $H_{\mathrm{M}}$ which satisfies (5), is given by $(7 b)$, whereas the zero-field Néel temperature $T_{\mathrm{N}}(0)$ is given by $(7 c)$; therefore :

$$
T_{\mathrm{N}}\left(H_{\mathrm{M}}\right) / T_{\mathrm{N}}(0)=\sqrt{2 C_{3} / C_{2}} .
$$

According to M.F.A.. the R.H.S. is equal to $\sqrt{2}$. and a field increases the Neel temperature by a maximum factor $\sqrt{2}$. The exact value is expected to be even larger because spin fluctuations. which decrease the M.F.A. value of $T_{\mathrm{N}}$. increase with $n$. so that $C_{n}$ is expected to increase when $n$ increases.

5. Ideal, two-dimensional antiferromagnets. 5.1 IDEAL HEISENBERG ANTIFERROMAGNET $(n=3)$. This system is believed to have no transition in 2 dimensions in zero field [6]. If a field $\mathbf{H}$ is applied. the system behaves like an XY model if (5) holds. The XY model has a transition without long range order [7] at $T_{0} \approx|J| s^{2} / K_{\mathrm{B}}$; therefore a transition without long range order is expected for the two-dimensional, Heisenberg model in a field. We speculate the transition temperature to be of order :

$$
K_{\mathrm{B}} T_{0} \approx H s
$$

5.2 IdEAL XY ANTIFERROMAGNET $(n=2)$. - It can be shown that the field is equivalent to an Ising anisotropy. and must produce long range order in the perpendicular direction below some temperature of order $|J| s^{2} / K_{\mathrm{B}}$.

These results are spectacular. but their applicability to real systems is problematic. as will be seen in the next section.

6. Real systems. - Real systems are not pure Heisenberg or XY but always have a small anisotropy ; if this anisotropy is of the Ising type. as is the case for most quasi-two-dimensional antiferromagnets [8], the anisotropy will dominate and the field will have a small effect. except above the spin-flop value which is usually very high. In principle. experiments above the spin-flop field would be very interesting because the two-dimensional character is much better in antiferromagnets than in ferromagnets. so that one might come rather close to the ideal two-dimensional, XY magnet. However. field heterogeneities can have a dramatic effect and such experiments are extremely difficult.

We now come back to TMMC. an easy-plane. quasi-one-dimensional antiferromagnet. We have some hope that our mechanism can account for the increase of $T_{N}$ for a field within the plane. but a quantitative calculation requires the consideration of the easy plane anisotropy and of the tetragonal anisotropy within the plane. The importance of the easyplane anisotropy results from the spectacular difference between $(2 b)$ and $(2 c)$. The tetragonal anisotropy is expected to be of some importance when it is larger than the field.

Appendix : Derivation of equation (6). - It is possible. by a decimation procedure. to change the (intrachain) inter-site distance from $l$ to $L$ by elimination of a fraction $(L-l) / L$ of the spin variables in the Hamiltonian (1).

$H$ will be assumed to be zero. since its effect is essentially to reduce $n$ by $l$. as shown in section 3 . In this case. $J$ can be assumed to be positive. If $H=0$, the change of scale does not modify the form of (1) if $L$ is not too large. and the new. effective coupling constants $\widetilde{J}_{\mathbf{L}}$ and $\tilde{J}_{\mathbf{L}}^{\prime}$ are given by :

$$
\begin{aligned}
\xi\left(\beta \tilde{J}_{\mathbf{L}}\right) & =\frac{1}{L} \xi(\beta J) \\
\tilde{J}_{\mathbf{L}}^{\prime} & =L J^{\prime}
\end{aligned}
$$

where $\xi$ is a well-defined function of $\beta J$. defined by (2). Equations (A. 1) and (A.2) hold if :

$$
\xi\left(\beta \tilde{J}_{\mathrm{L}}\right) \gg 1
$$

and :

$$
\beta \tilde{J_{\mathbf{L}}^{\prime}} \ll 1
$$

Consider all systems which can be scaled in such a way that $\xi\left(\beta J_{\mathrm{L}}\right)=\xi_{0}$ has a given value which corresponds to a given value $\beta_{0} J_{0}$ of $\beta J$. The probability distribution only depends on $\beta \tilde{J}_{\mathbf{L}}^{\prime} s^{2}$. which, according to (A.1. 2). is equal to :

$$
\beta \tilde{J}_{\mathbf{L}}^{\prime} s^{2}=\beta L J^{\prime} s^{2}=\beta s^{2} J^{\prime} \xi(\beta J) / \xi_{0} .
$$

Since all long wavelength properties are described by this unique parameter. three-dimensional order appears for a well-defined value of this quantity. and equation (6) follows. Since $\xi_{0}$ is arbitrary, this result is general. 


\section{References}

[1] Dupas, C., Renard, J. P., Solid State Commun. 20 (1976) 581.

[2] Imry, Y., Pincus, P., Scalapino, D., Phys. Rev. B 12 (1975) 1978.

[3] See, for instance, Steiner, M., Villain, J., Windsor, C. G., Adv. Phys. 25 (1976) 87 and references therein.
[4] Morita, T., Horiguchi, T., Physica 83A (1975) 519.

[5] Lovesey, S. W., Loveluck, J. M., J. Phys. C 9 (1976) 3639.

[6] Brezin, E., Zinn-Justin, J., Phys. Rev. Lett. 36 (1976) 691.

[7] Kosterlitz, J. M., Thouless, D. J., J. Phys. C 6 (1973) 1181.

[8] De Jongh, L. J., Miedema, A. R., Adv. Phys. 23 (1974) 1. 